\title{
Advanced Robotics
}

\section{Robust adaptive multi-task tracking control of redundant manipulators with dynamic and kinematic uncertainties and unknown disturbances}

\author{
Yi Ren, Yang Zhou, Yechao Liu, Yikun Gu, Minghe Jin \& Hong Liu
}

To cite this article: Yi Ren, Yang Zhou, Yechao Liu, Yikun Gu, Minghe Jin \& Hong Liu (2017): Robust adaptive multi-task tracking control of redundant manipulators with dynamic and kinematic uncertainties and unknown disturbances, Advanced Robotics, DOI: 10.1080/01691864.2016.1277553

To link to this article: http://dx.doi.org/10.1080/01691864.2016.1277553

曲 Published online: 28 Jan 2017.

Submit your article to this journal $\widetilde{ }$

View related articles $\asymp$

View Crossmark data \ 
FULL PAPER

\title{
Robust adaptive multi-task tracking control of redundant manipulators with dynamic and kinematic uncertainties and unknown disturbances
}

\author{
Yi Rena, Yang Zhou ${ }^{b}$, Yechao Liu ${ }^{a}$, Yikun Gua ${ }^{a}$ Minghe Jin a and Hong Liu ${ }^{a}$

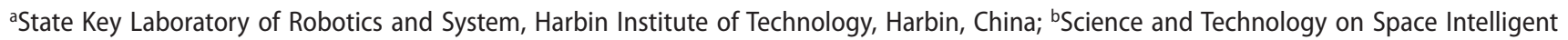 \\ Control Laboratory, Beijing Institute of Control Engineering, Beijing, China
}

ARTICLE HISTORY Received 25 June 2016; Revised 31 October 2016; Accepted 20 December 2016

KEYWORDS Robust adaptive control; multi-task tracking; redundant manipulators; uncertain kinematics and dynamics

\section{Introduction}

With task-oriented strategy and the aid of their eyes, human beings can skillfully grasp an unknown tool to execute different tasks without exact knowledge of the kinematics and dynamics of their arms and the tool. Advanced humanoid robots, like Atlas, Justin [1], Robonaut [2] or the HIT-Robonaut as shown in Figure 1, are often equipped with robot vision system and redundant manipulators to imitate human behavior and control strategy. The robot vision system can provide real-time feedback of environmental information, and the redundant manipulator can execute multiple tasks simultaneously. By fusing the vision information with the encoder signals of manipulators, it is possible to greatly improve the flexibility of the robot.

When the redundant manipulator grasps an unknown welding pistol to execute multiple tasks, such as welding in preassigned shape and simultaneously maintaining a fixed pose of the welding rod relative to welded parts, dynamic and kinematic uncertainties (including the unknown grasp matrix) and unknown nonlinearities may greatly degrade the performance of the system if without any adaptability in its controller.

To maintain high tracking performance when dynamic uncertainty exists in the redundant manipulator, adaptive control and robust control $[3,4]$ have been proposed. Compared with the latter one, adaptive control is more suitable for coping with large load variations due to its ability to hold the promise of uniform performance. Based on the differences in driving signals of the parameter adaptation mechanism, adaptive controllers can be classified into three categories: direct [5], indirect [6] and composite [7-9] adaptive controllers. Compared with the other two, composite adaptive controllers extract parameter information from both the prediction error and tracking error, thus leading to a faster convergence rate and better tracking accuracy [10].

Until the past decade, globally convergent adaptive controllers have been proposed to simultaneously deal with the uncertain kinematics and dynamics. In the view of controller design philosophy, they can be generally classified into two categories: passivity-based control [11-14] and inverse dynamic control (computed-torque control) $[15,16]$. Although the inverse dynamics control approach, as stated in Ref. [15], can guarantee a uniform performance of the manipulator over the entire workspace, the passivity-based control is easier to implement owing to its attractive properties, i.e. requirement of the joint acceleration and inverse of the estimated inertial matrix can be exempted. The basic design philosophy of passivity-based controller is to reshape the system's natural energy such that the control objective can be achieved. However, most of these adaptive Jacobian controllers aim at non-redundant manipulators.

Although the adaptive controllers in Refs. [12,14] are universal for redundant and non-redundant manipulators, multi-task tracking capability cannot be obtained. To accomplish multi-task tracking with concurrent adaptation to both dynamic and kinematic uncertainties is much different compared with these controllers. Potential algorithmic singularity during the adaptation may lead to a complicated coupling and conflict among the multiple tasks [17].

How to appropriately deal with the multi-task tracking needs a detailed discussion. Recently, several adaptive controllers have been specially designed for multi-task

CONTACT YechaoLiu $\otimes$ yechaohit@hit.edu.cn

Please see video abstract here.

(c) 2017 Taylor \& Francis and The Robotics Society of Japan 


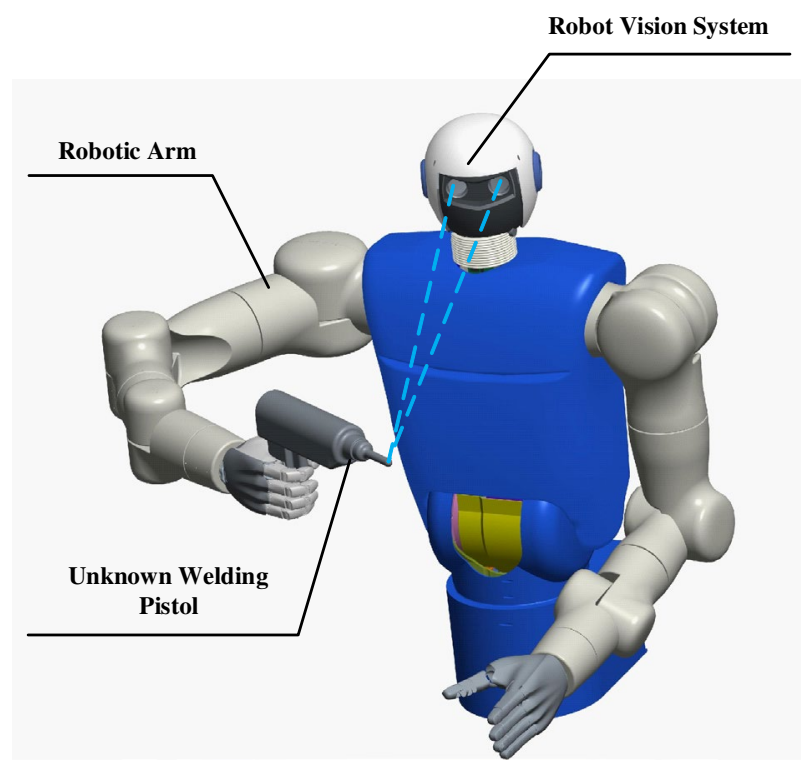

Figure 1. HIT-Robonaut system with unknown tool.

tracking. In Ref. [18], Enver Tatlicioglu et al. utilized Lyapunov-based analysis to design an adaptive controller with which the asymptotical tracking in the task space and subtask objective are simultaneously achieved when dynamic uncertainty exists. Then Sedgehian et al. [19] successfully extended the adaptive control to multi-priority control framework. The asymptotical stability and convergence in tracking error of the main task and subtask are both guaranteed according to the allocated priority. However, the kinematics and task Jacobians are assumed to be exactly known in both of these two controllers. The adaptive controller scheme in Ref. [19] only utilizes the tracking error of the primary task for dynamic parameter adaptation, large gain is needed when facing severe uncertainties, which may induce oscillating of the estimated parameters or even cause the whole system unstable. Moreover, convergences of all the tasks are sequential according to their corresponding priority levels [20], namely, convergence of the task with lower priority cannot be achieved until all the tasks with higher priority converge even when the prioritized tasks are independent. This is inefficient especially for the multitask tracking with many task layers.

Furthermore, it should be noted that most of the research works mentioned above are based on the assumption that the robotic arms are free of unknown nonlinearities. However, this is too restrictive since unknown nonlinear dynamics, such as friction, backlash or deadzone are ubiquitous phenomena in the manipulators' joint [21]. With detailed characteristic of these nonlinearities, model-based schemes are preferred for compensation [22]. But no matter which kinds of corresponding models are used, the unstructured modeling errors always exist and would deteriorate the system performance.
Hence in this paper, we propose a novel robust adaptive multi-priority controller (RAM) for redundant manipulator to address the prioritized multi-task tracking problem when dynamic/kinematic uncertainties and unknown disturbances exist. A strategy is established to comprehensively utilize multi-task tracking information to accelerate the concurrent adaptation of kinematics and dynamics. According to the condition of algorithmic singularity, we discuss and design the controller in independent case and dependent case, respectively. The modified controller for the second case holds universality whether facing algorithmic singularity or not.

\section{Problem formulation}

\subsection{Robot dynamics and kinematics}

When using model-based compensator as feedforward to cope with nonlinear dynamics such as friction and backlash, residuals of unstructured modeling errors or unparameterizable parts can be defined as:

$$
\tau_{d}=\left(\tau_{f}-{ }^{m} \tau_{f}\right)+\left(\tau_{b}-{ }^{m} \tau_{b}\right)
$$

where $\tau_{f}$ and $\tau_{b}$ denote the joint friction and the backlash existing in the transmission gear, respectively, ${ }^{m} \tau_{f}$ and ${ }^{m} \tau_{b}$ represent their corresponding model-based compensator.

Then the dynamics of an $\mathrm{n}$-link rigid redundant manipulator with the unknown tool considered as part of the last link can be expressed as:

$$
M(q) \ddot{q}+C(q, \dot{q}) \dot{q}+g(q)=\tau+\tau_{d}
$$

where $q \in R^{n}$ is the joint angle vector, $M(q) \in R^{n \times n}$ is the inertial matrix, $C(q, \dot{q}) \dot{q} \in R^{n}$ is the vector of centripetal and Coriolis torques, $g(q) \in R^{n}$ is the vector of gravitational torques $\tau \in R^{n}$ is the exerted joint torque, $\tau_{d} \in R^{n}$ denotes bounded unstructured modeling uncertainties or exogenous disturbances. Several essential properties of the manipulator dynamics (2) facilitate the adaptive control design even if this dynamic equation is complex and highly nonlinear.

Property 1 . The inertial matrix $M(q)$ is uniformly positive definite and symmetric [23].

Property 2. $(\dot{M}(q)-2 C)$ is skew-symmetric so that $v^{T}(\dot{M}(q)-2 C) v=0$ for all $v \in R^{n}$.

Property 3. The manipulator dynamics (2) is linear in a set of physical parameters $\theta_{d}=\left[\theta_{d 1}, \theta_{d 2}, \ldots, \theta_{d p}\right]^{T}$.

$$
M(q) \ddot{q}+C(q, \dot{q}) \dot{q}+g(q)=Y_{d}(q, \dot{q}, \ddot{q}) \theta_{d}
$$

where the dynamic regression matrix $Y_{d}(\cdot) \in R^{n \times p}$ is bounded for bounded argument signals.

Let $x_{t} \in R^{m}$ be a task space vector defined by

$$
x_{t}=h(q)
$$


where $m<n$ for redundant manipulators, $h(\cdot) \in R^{n} \rightarrow R^{m}$ is a transformation describing the relation between the joint space and task space. The task-space velocity $\dot{x}_{t}$ is related to joint-space velocity $\dot{q}$ through the Jacobian matrix and can be expressed linearly in a set of kinematic parameters $\theta_{k}=\left[\theta_{1}, \theta_{2}, \ldots, \theta_{q}\right]^{T}$, such as joint offsets and link lengths.

$$
\dot{x}_{t}=J_{t}(q) \dot{q}=Y_{k t}(q, \dot{q}) \theta_{k}
$$

where $J_{t}(q) \in R^{m \times n}$ is manipulator Jacobian and $Y_{k t}(\cdot) \in R^{m \times q}$ denotes the kinematic regression matrix.

\subsection{Multi-priority inverse kinematics of redundant manipulator}

For redundant manipulators, multiple tasks can be arranged in priority to try to fulfill most of them, hopefully all of them. Multi-priority control is a framework to achieve this goal by proper task-priority assignment [24]. To simplify the discussion, the problem for a case with two prioritized task is formulated. Extending to the case with multiple tasks is easy to implement by recursive method [25]. The primary task with the first priority is specified by the first manipulation variable $x_{1} \in R^{m_{1}}\left(m_{1}<n\right)$ and the subtask with the second priority by $x_{2} \in R^{m_{2}}\left(m_{2}<n\right)$.

$$
\dot{x}_{i}=J_{i}(q) \dot{q}
$$

where $J_{i} \in \mathrm{R}^{m_{i} \times n}(i=1,2)$ denotes the Jacobian matrix of the $i$-th task. To derive the adaptive controller in a compact form, we define $\dot{x}=\left[\dot{x}_{1}^{T}, \dot{x}_{2}^{T}\right]^{T}$ as the task velocity vector and the following equation can be obtained:

$$
\dot{x}=J \dot{q}=Y_{k} \theta_{k}
$$

where $J=\left[J_{1}^{T}, J_{2}^{T}\right]^{T} \in R^{\left(m_{1}+m_{2}\right) \times n}, Y_{k}=\left[Y_{k 1}^{T}, Y_{k 2}^{T}\right]^{T} \in R^{\left(m_{1}+m_{2}\right) \times q}$. $Y_{k i} \in R^{m_{i} \times q}$ denotes the kinematic regression of the $i$-th task.

Since the joint space of the redundant manipulator is governed by multiple tasks, then the inverse kinematic solution considering task priority can be given by [24]

$$
\dot{q}_{r}=J_{1}^{\dagger} \dot{x}_{1 r}+N_{1} \bar{J}_{2}^{\dagger}\left(\dot{x}_{2 r}-J_{2} J_{1}^{\dagger} \dot{x}_{1 r}\right)
$$

where $\dot{q}_{r}$ denotes the reference joint velocity, $\dot{x}_{1 r} \in R^{m_{1}}$ and $\dot{x}_{2 r} \in R^{m_{2}}$ are the reference velocity for the primary task and subtask, respectively. They are defined as $\dot{x}_{i r}=\dot{x}_{i d}+\alpha \tilde{x}_{i}$ where $\alpha>0$ is an appropriate positive constant and $\tilde{x}_{i}=x_{i d}-x_{i}$ denotes the task tracking error for $i$-th task, the index $d$ stands for the desired trajectory. $(A)^{\dagger}=A^{T}\left(A A^{T}\right)^{-1}$ represents the pseudoinverse of the related matrix $A$ and $N_{1}=\left(I-J_{1}^{\dagger} J_{1}\right) \in R^{n \times n}$ is the corresponding null-space projector for $J_{1} . \bar{J}_{2}=J_{2} N_{1}$ is the so-called projected Jacobian, which determines the available range for the execution of the subtask without affecting the primary task.
With the above redundancy resolution, correct primary-task solution is expected as long as the sole primary-task Jacobian matrix is full-rank. Multiplying both sides of (8) by $J_{1}$ leads to following equation when $\dot{q} \rightarrow \dot{q}_{r}$.

$$
\dot{\tilde{x}}_{1}+\alpha \tilde{x}_{1}=0
$$

Before the discussion of the performance of subtask, two definitions concerning the relationship between the two tasks should be given [26]

The two tasks will be defined as independent if

$$
\rho\left(J_{1}^{\dagger}\right)+\rho\left(J_{2}^{\dagger}\right)=\rho\left(\left[\begin{array}{ll}
J_{1}^{\dagger} & J_{2}^{\dagger}
\end{array}\right]\right)
$$

where $\rho(\cdot)$ denotes the rank of the related matrix.

They will be defined as dependent if

$$
\rho\left(J_{1}^{\dagger}\right)+\rho\left(J_{2}^{\dagger}\right)>\rho\left(\left[\begin{array}{ll}
J_{1}^{\dagger} & J_{2}^{\dagger}
\end{array}\right]\right)
$$

Independence condition guarantees the full rank of $\bar{J}_{2}$ and the avoidance of algorithmic singularity. Then the subtask can be expected to perform completely. While dependence case results in rank deficiency of $\bar{J}_{2}$, which further affects the tracking performance of subtask. This is because ill-conditioned and discontinuous joint velocity solutions are experienced when approaching algorithmic singularity.

\section{Composite adaptive controller}

In this section, we will incorporate a passivity-based adaptive Jacobian controller into the multi-priority framework for redundant manipulators to solve the tracking problem brought by uncertain kinematics and dynamics. In this composite adaptive controller, task-space motion tracking error and prediction error are both used to drive the parameter estimation. Furthermore, two different cases are discussed, respectively, according to the relationship of the two generic tasks (dependence and independence). The redundant manipulator is required to simultaneously track two desired prioritized task trajectories $x_{i d}$ for $(i=1,2)$ and it is assumed that $J_{i}$ is globally non-singular and $x_{i d}, \dot{x}_{i d}, \ddot{x}_{i d}$ are all bounded.

\subsection{Independent case}

In this case, the two tasks are assigned to be independent and the estimated Jacobians $\left(\hat{J}_{1}\right.$ and $\left.\hat{J}_{2}\right)$ also uniformly satisfy the independence condition.

Since joint accelerations $\ddot{q}$ and the task space velocity $\dot{x}$ are generally sensitive to noise, direct measurement of these two signals should be avoided.

Convolving both sides of (2) by the impulse response of a low-pass filter yields [27]: 


$$
\begin{aligned}
y_{d} & =\int_{0}^{t} \omega(t-r) \tau \mathrm{d} r \\
& =\int_{0}^{t} \omega(t-r)[M(q) \ddot{q}+C(q, \dot{q}) \dot{q}+g(q)] \mathrm{d} r
\end{aligned}
$$

which we can be written as:

$$
y_{d}(t)=W_{d}(q, \dot{q}, t) \theta_{d}
$$

where $\omega(t)$ is the impulse response of the filter $\lambda_{f} /\left(\lambda_{f}+p\right)$ and $y_{d}$ is the filtered joint torque, $\lambda_{f}>0$ is the filter parameter and $p$ is the Laplace operator. The filter is exponentially stable and strictly proper.

Measurement of task-space velocity can be avoided similarly by filtering Equation (7)

$$
y_{k i}=\frac{\lambda_{f k i}}{\lambda_{f k i}+p} \dot{x}=W_{k i}(t) \theta_{k} \quad(\text { for } i=1,2)
$$

where $\lambda_{f k i}>0$ and $y_{k_{i}}$ is the filtered task-space velocity.

\subsubsection{Design of the controller}

In the presence of uncertainties, the dynamic and kinematic parameters are uncertain. Let $\hat{\theta}_{d}$ and $\hat{\theta}_{k}$ be the estimate of the $\theta_{d}$ and $\theta_{k}$, then the estimate of the filtered torque $\hat{y}_{d}(t)$ can be expressed as:

$$
\hat{y}_{d}(t)=W_{d}(q, \dot{q}, t) \hat{\theta}_{d}
$$

and the task-space velocity $\hat{\dot{x}}$ as:

$\hat{\dot{x}}=\left(\begin{array}{c}\hat{x}_{1} \\ \hat{\dot{x}}_{2}\end{array}\right)=\left(\begin{array}{c}\hat{J}_{1} \\ \hat{J}_{2}\end{array}\right) \dot{q}=\hat{J} \dot{q}=\left(\begin{array}{c}Y_{k 1} \\ Y_{k 2}\end{array}\right) \hat{\theta}_{k}=Y_{k} \hat{\theta}_{k}$

Combining with Equation (14), the estimate of the filtered velocity $\hat{y}_{k}$ can be given as:

$$
\hat{y}_{k}=W_{k}(t) \hat{\theta}_{k}
$$

Differentiating Equation (16) with respect to time leads to:

$$
\hat{\ddot{x}}=\hat{J} \ddot{q}+\dot{\hat{J}} \dot{q}
$$

where $\hat{x}$ is the time derivative of $\hat{x}$.

Now define a joint-space sliding variable $s_{q} \in R^{n}$ as:

$$
s_{q}=\dot{q}_{r}-\dot{q}
$$

Its time derivative can be given as:

$$
\dot{s}_{q}=\ddot{q}_{r}-\ddot{q}
$$

where $\dot{q}_{r}$ expressed by Equation (8) should be rewritten as follows when considering the kinematic uncertainties

$$
\dot{q}_{r}=\hat{J}_{1}^{\dagger} \dot{x}_{1 r}+\bar{N}\left(\dot{x}_{2 r}-\hat{J}_{2} \hat{J}_{1}^{\dagger} \dot{x}_{1 r}\right)
$$

where $\bar{N}=\hat{N}_{1}\left(\hat{J}_{2} \hat{N}_{1}\right)^{\dagger}$ and $(\hat{\mathrm{A}})$ is the estimated form of the related matrix $\mathrm{A}$.

To eliminate the terms of the task-space velocities in $\ddot{q}_{r}$, $\ddot{x}_{1 r}$ and $\ddot{x}_{2 r}$ should be replaced by their corresponding estimated terms, then the following expression can be given:

$$
\begin{aligned}
\hat{\ddot{q}}_{r}= & \hat{J}_{1}^{\dagger} \hat{\ddot{x}}_{1 r}+\dot{\hat{J}}_{1}^{\dagger} \dot{x}_{1 r} \dot{\bar{N}}\left(\dot{x}_{2 r}-\hat{J}_{2} \hat{J}_{1}^{\dagger} \dot{x}_{1 r}\right) \\
& +\bar{N}\left(\hat{\ddot{x}}_{2 r}-\hat{\hat{J}}_{2} \hat{J}_{1}^{\dagger} \dot{x}_{1 r}-\hat{J}_{2} \dot{\hat{J}}_{1}^{\dagger} \dot{x}_{1 r}-\hat{J}_{2} \hat{J}_{1}^{\dagger} \hat{\ddot{x}}_{1 r}\right) \\
= & \ddot{q}_{r}+\alpha\left(\hat{J}_{1}^{\dagger}-\bar{N} \hat{J}_{2} \hat{J}_{1}^{\dagger}\right)\left(\dot{x}_{1}-\hat{\dot{x}}_{1}\right)+\alpha \bar{N}\left(\dot{x}_{2}-\hat{\dot{x}}_{2}\right) \\
= & \ddot{q}_{r}+\tilde{\beta}
\end{aligned}
$$

where $\quad \hat{\dot{x}}_{i r}=\ddot{x}_{i r}+\alpha\left(\dot{x}_{i}-\hat{\dot{x}}_{i}\right)$ for $(i=1,2)$, $\tilde{\beta}=\alpha\left[\hat{J}_{1}^{\dagger}-\bar{N} \hat{J}_{2} \hat{J}_{1}^{\dagger}, \bar{N}\right] Y_{k}\left(\theta_{k}-\hat{\theta}_{k}\right)$.

Under the assumption that the two estimated Jacobian always satisfy the independence condition (10), then using $\hat{J}$ to multiply both sides of (21), we can obtain:

$$
\hat{J} \dot{q}_{r}=\left[\begin{array}{l}
\hat{J}_{1} \\
\hat{J}_{2}
\end{array}\right] \quad \dot{q}_{r}=\left[\begin{array}{c}
\dot{x}_{1 r} \\
\dot{x}_{2 r}
\end{array}\right]
$$

Next, define an estimated task-space sliding variable $\hat{s}_{x}=\left[\begin{array}{cc}\hat{s}_{1 x}^{T} & \hat{s}_{2 x}^{T}\end{array}\right]^{T} \in R^{\left(m_{1}+m_{2}\right) \times 1}$

$$
\hat{s}_{x}=\dot{x}_{r}-\hat{\dot{x}}=\Delta \hat{\dot{x}}+\alpha \tilde{x}
$$

where $\dot{x}_{r}=\left[\begin{array}{cc}\dot{x}_{1 r}^{T} & \dot{x}_{2 r}^{T}\end{array}\right]^{T}$ is the task-space reference velocity, $\Delta \hat{\dot{x}}=\left[\begin{array}{cc}\Delta \hat{\dot{x}}_{1}^{T} & \Delta \hat{\dot{x}}_{2}^{T}\end{array}\right]^{T}$ and $\tilde{x}=\left[\begin{array}{cc}\tilde{x}_{1}^{T} & \tilde{x}_{2}^{T}\end{array}\right]^{T}$ with $\Delta \hat{\dot{x}}_{i}=\dot{x}_{i d}-\hat{\dot{x}}_{i}$.

Combining (16), (19), (23) and (24) yields:

$$
\hat{J} s_{q}=\hat{s}_{x}
$$

Also, to extract parameter information from the prediction error, we define the prediction error of the filtered torque as:

$$
e_{d}=\hat{y}_{d}-y_{d}=W_{d}(q, \dot{q}, t) \tilde{\theta}_{d}
$$

and the prediction error of the filtered task-space velocity as:

$$
e_{k}=\hat{y}_{k}-y_{k}=W_{k}(t) \tilde{\theta}_{k}
$$

where $W_{k}(t)=\left[\begin{array}{ll}W_{k 1}^{T}(t) & W_{k 2}^{T}(t)\end{array}\right]^{T}, \tilde{\theta}_{d}=\hat{\theta}_{d}-\theta_{d}$ and $\tilde{\theta}_{k}=\hat{\theta}_{k}-\theta_{k}$ are the dynamic and kinematic parameter prediction error, respectively.

Using Property 1 , the following linearly parameterized equation associated with the dynamics model (2) can be obtained:

$$
M(q) \hat{\ddot{q}}_{r}+C(q, \dot{q}) \dot{q}_{r}+g(q)=Y_{\mathrm{d} r}\left(q, \dot{q}, \dot{q}_{r}, \hat{\ddot{q}}_{r}\right) \theta_{d}
$$


where the joint acceleration and task velocity in $Y_{d}(q, \dot{q}, \ddot{q})$ are eliminated. Then the estimated form of (28) is written as:

$$
\hat{M}(q) \hat{\ddot{q}}_{r}+\hat{C}(q, \dot{q}) \dot{q}_{r}+\hat{g}(q)=Y_{\mathrm{d} r}\left(q, \dot{q}, \dot{q}_{r}, \hat{\ddot{q}}_{r}\right) \hat{\theta}_{d}
$$

Substituting (22) into (28) results in:

$$
M(q) \ddot{q}_{r}+C(q, \dot{q}) \dot{q}_{r}+g(q)=Y_{\mathrm{d} r}\left(q, \dot{q}, \dot{q}_{r}, \hat{\ddot{q}}_{r}\right) \theta_{d}-M \tilde{\beta}
$$

where $M \tilde{\beta}$ is linear in a new set of coupling parameters $\theta_{d k}$. Hence (30) can be rewritten as:

$$
\begin{aligned}
& M(q) \ddot{q}_{r}+C(q, \dot{q}) \dot{q}_{r}+g(q) \\
& =Y_{\mathrm{d} r}\left(q, \dot{q}, \dot{q}_{r}, \hat{\ddot{q}}_{r}\right) \theta_{d}-\hat{Y}_{\mathrm{d} r}\left(q, \dot{q}, \dot{q}_{r}, \hat{\theta}_{k}\right) \theta_{\mathrm{d} k}
\end{aligned}
$$

Combining (31) with (2) leads to

$$
M \dot{s}_{q}+C s_{q}=Y_{\mathrm{d} r} \theta_{d}-\hat{Y}_{\mathrm{d} r} \theta_{\mathrm{d} k}-\tau-\tau_{d}
$$

Based on the above error analysis, the following adaptive controller is proposed:

$$
\tau=Y_{\mathrm{d} r} \hat{\theta}_{d}-\hat{Y}_{\mathrm{d} r} \hat{\theta}_{\mathrm{d} k}+\hat{J}^{T} K_{m} \hat{s}_{x}+K_{p} s_{q}+\Lambda
$$

where $K_{m} \in R^{\left(m_{1}+m_{2}\right) \times\left(m_{1}+m_{2}\right)}$ and $K_{p} \in R^{n \times n}$ are both positive-definite symmetric matrix. $\Lambda=\left[\Lambda_{1}, \Lambda_{2} \ldots \Lambda_{n}\right]^{T} \in R^{n}$ denotes the adaptive robust compensator which is presented as

$$
\Lambda_{i}=\hat{D}_{i} \tanh \left(s_{q i} / \varsigma\right)
$$

where $\hat{D}_{i}$ is the estimate of the $D_{i}$ 's upper bound, and $\varsigma$ is a positive constant to regulate the smoothness of the switch function. $s_{q i}$ denotes the $i$-th element of the sliding vector $s_{q} . D_{i}$ is defined as the supremum of the unstructured part

$$
D_{i}=\sup \left(\left|\tau_{d i}\right|\right)
$$

where $\tau_{d i}$ denotes the $i$-th element of the unstructured vector.

Remark 1: In (34), tangent hyperbolic functions are chosen as the switch function in the robust compensator rather than the traditional sign function to avoid the discontinuity and chattering. Moreover, the upper bound is adaptively regulated since it cannot be exactly known in advance and a conservative value often leads to severe chattering.

The parameter estimates $\hat{\theta}_{d}, \hat{\theta}_{\mathrm{d} k}$ and $\hat{\theta}_{k}$ are updated by,

$$
\dot{\hat{\theta}}_{d}=P_{d}(t)\left(Y_{\mathrm{d} r}^{T} s_{q}-W_{d}^{T} R_{d} e_{d}\right)
$$

$$
\begin{gathered}
\dot{\hat{\theta}}_{\mathrm{d} k}=-L_{\mathrm{d} k} \hat{Y}_{\mathrm{d} r}^{T} s_{q}-\Im \dot{\theta}_{\mathrm{d} k} \\
\dot{\hat{\theta}}_{k}=P_{k}(t)\left(-2 \alpha Y_{k}^{T} K_{m} \tilde{x}-W_{k}^{T} R_{k} e_{k}\right) \\
\dot{\hat{D}}_{i}=L_{r} s_{q i} \tanh \left(s_{q i} / \varsigma\right)-\aleph \hat{D}_{i}
\end{gathered}
$$

where $R_{d} \in R^{n \times n}$ and $R_{k} \in R^{\left(m_{1}+m_{2}\right) \times\left(m_{1}+m_{2}\right)}$ are corresponding weighting matrices indicating the weights of the prediction error in adaptation laws. Both of them are uniformly positive definite. $L_{d k}, \mathfrak{\Im}, L_{r}$ and $\aleph$ are all positive constant gain.

Remark 2: When the two tasks are independent, their tracking errors are both used in (33)-(38) and together accelerate the parameter estimation. In fact, $\hat{J}, \hat{s}_{x}, \tilde{x}$ and $W_{k}^{T} R_{k} e_{k}$ in the above control law and updating laws can be replaced by $\hat{J}_{1}, \hat{s}_{x 1}, \tilde{x}_{1}$ and $W_{k 1}^{T} R_{k 1} e_{k 1}$ since the primary task can always be performed completely with the highest priority when considering (21). The stability and convergence of the subtask tracking can be guaranteed conditionally to the set $A=\left\{\tilde{x}_{1}, s_{q}, \tilde{x}_{2} \mid \tilde{x}_{1}=0, s_{q}=0\right\}$ [19]. But in the sense of achieving high performance of the subtask as the primary one when they are independent, the controller (33)-(39) outperforms the aforementioned one with which the convergence of the subtask only can be stated after the convergence of the primary task is fulfilled [28].

To eliminate the drawbacks of slow or oscillatory convergence in gradient estimator and the gain vanishing/explosion in standard least-square estimator, bounded-gain-forgetting (BGF) estimator is used since it can guarantee the uniformly boundedness of the estimated parameters and gains in the absence of persistent excitation.

Then the gain update equations for $P_{d}$ and $P_{k}$ are given as:

$$
\begin{gathered}
\frac{d}{\mathrm{~d} t}\left[P_{d}\right]=\lambda_{d}(t) P_{d}-P_{d} W_{d}^{T}(t) R_{d} W_{d}(t) P_{d} \\
\lambda_{d}(t)=\lambda_{0}\left(1-\left\|P_{d}\right\| / k_{0}\right) \\
\frac{d}{\mathrm{~d} t}\left[P_{k}\right]=\lambda_{k}(t) P_{k}-P_{k} W_{k}^{T}(t) R_{k} W_{k}(t) P_{k} \\
\lambda_{k}(t)=\lambda_{0 k}\left(1-\left\|P_{k}\right\| / k_{0 k}\right)
\end{gathered}
$$

where $\lambda_{0}\left(\lambda_{0 k}\right)$ and $k_{0}\left(k_{0 k}\right)$ are all positive constants representing the maximum forgetting rates and pre-specified bounds for the norm of gain matrix, respectively. 


\subsubsection{Stability and convergence analysis}

Before the Lyapunov analysis, three important lemmas should be first stated

Lemma 1: The following inequality always holds for any $u \in R$ and $\kappa>0$

$$
0 \leq|u|-u \tanh \left(\frac{u}{\varsigma}\right) \leq \delta \varsigma, \quad \delta=0.2785
$$

Lemma 2 [29]: If $f, \dot{f} \in L_{\infty}$ and $f \in L_{p}$ for some $p \in[1, \infty)$, then $f(t) \rightarrow 0$ as $t \rightarrow \infty$.

Lemma 3: (Young's inequality) $\forall(x, y) \in R^{2}$, the following inequality always holds:

$$
x y \leq \frac{\varepsilon^{p}}{p}|x|^{p}+\frac{1}{q \varepsilon^{q}}|y|^{q}
$$

where $\varepsilon>0, p>1, q>1$ and $(p-1)(q-1)=1$.

$$
\begin{aligned}
\dot{V}= & s_{q}^{T}\left(-Y_{\mathrm{d} r} \tilde{\theta}_{d}+\hat{Y}_{\mathrm{d} r} \tilde{\theta}_{\mathrm{d} k}-\hat{J}^{T} K_{m} \hat{s}_{x}-K_{p} s_{q}-\Lambda-\tau_{d}\right) \\
& +\tilde{\theta}_{d}^{T}\left(Y_{\mathrm{d} r}^{T} s_{q}-W_{d}^{T} R_{d} e_{d}\right)-\frac{1}{2} \tilde{\theta}_{d}^{T} \lambda_{d} P_{d}^{-1} \tilde{\theta}_{d} \\
& +\frac{1}{2} \tilde{\theta}_{d}^{T} W_{d}^{T} R_{d} W_{d} \tilde{\theta}_{d}-\tilde{\theta}_{\mathrm{d} k}^{T}\left(\hat{Y}_{\mathrm{d} r}^{T} s_{q}+L_{\mathrm{d} k}^{-1} \Im \hat{\theta}_{\mathrm{d} k}\right) \\
& -\frac{1}{2} \tilde{\theta}_{k}^{T} \lambda_{k} P_{k}^{-1} \tilde{\theta}_{k}+\frac{1}{2} \tilde{\theta}_{k}^{T} W_{k}^{T} R_{k} W_{k} \tilde{\theta}_{k} \\
& -2 \alpha \tilde{\theta}_{k}^{T} Y_{k}^{T} K_{m} \tilde{x}-\tilde{\theta}_{k}^{T} W_{k}^{T} R_{k} e_{k}+2 \alpha \tilde{x}^{T} K_{m} \dot{\tilde{x}} \\
& +\sum_{i=1}^{n} \tilde{D}_{i}^{T}\left(s_{q i} \tanh \left(s_{q i} / \varsigma\right)-L_{r}^{-1} \aleph \hat{D}_{i}\right) \\
= & -s_{q}^{T} K_{p} s_{q}-\frac{1}{2} \tilde{\theta}_{d}^{T} W_{d}^{T} R_{d} W_{d} \tilde{\theta}_{d}-\frac{\lambda_{d}}{2} \tilde{\theta}_{d}^{T} P_{d}^{-1} \tilde{\theta}_{d} \\
& -\frac{\lambda_{k}}{2} \tilde{\theta}_{k}^{T} P_{k}^{-1} \tilde{\theta}_{k}-\frac{1}{2} \tilde{\theta}_{k}^{T} W_{k}^{T} R_{k} W_{k} \tilde{\theta}_{k} \\
& -s_{q}^{T}\left(\Lambda+\tau_{d}\right)+\sum_{i=1}^{n}\left(\tilde{D}_{i}^{T} s_{q i} \tanh \left(s_{q i} / \varsigma\right)-\tilde{D}_{i}^{T} L_{r}^{-1} \aleph \hat{D}_{i}\right) \\
& -\left(\hat{J}_{s_{q}}\right)^{T} K_{m} \hat{s}_{x}-2 \alpha \tilde{\theta}_{k}^{T} Y_{k}^{T} K_{m} \tilde{x}+2 \alpha \tilde{x}^{T} K_{m} \dot{\tilde{x}}-\tilde{\theta}_{\mathrm{d} k}^{T} L_{\mathrm{d} k}^{-1} \widetilde{\Im} \hat{\theta}_{\mathrm{d} k} \\
& =\dot{V}_{1}+\dot{V}_{2}+\dot{V}_{3}+\dot{V}_{4}
\end{aligned}
$$

where

$$
\left\{\begin{array}{l}
\dot{V}_{1}=-s_{q}^{T} K_{p} s_{q}-\frac{1}{2} \tilde{\theta}_{d}^{T} W_{d}^{T} R_{d} W_{d} \tilde{\theta}_{d}-\frac{\lambda_{d}}{2} \tilde{\theta}_{d}^{T} P_{d}^{-1} \tilde{\theta}_{d}-\frac{\lambda_{k}}{2} \tilde{\theta}_{k}^{T} P_{k}^{-1} \tilde{\theta}_{k}-\frac{1}{2} \tilde{\theta}_{k}^{T} W_{k}^{T} R_{k} W_{k} \tilde{\theta}_{k} \\
\dot{V}_{2}=-s_{q}^{T}\left(\Lambda+\tau_{d}\right)+\sum_{i=1}^{n}\left(\tilde{D}_{i}^{T} s_{q i} \tanh \left(s_{q i} / \varsigma\right)-\tilde{D}_{i}^{T} L_{r}^{-1} \aleph \hat{D}_{i}\right) \\
\dot{V}_{3}=-\left(\hat{J} s_{q}\right)^{T} K_{m} \hat{s}_{x}-2 \alpha \tilde{\theta}_{k}^{T} Y_{k}^{T} K_{m} \tilde{x}+2 \alpha \tilde{x}^{T} K_{m} \dot{\tilde{x}}^{x} \\
\dot{V}_{4}=-\tilde{\theta}_{\mathrm{d} k}^{T} L_{\mathrm{d} k}^{-1} \Im \hat{\theta}_{\mathrm{d} k}
\end{array}\right.
$$

Then substituting the controller (33) into (32) with the definition of $\tilde{\theta}_{\mathrm{d} k}=\hat{\theta}_{\mathrm{d} k}-\theta_{\mathrm{d} k}$ yields the following dynamics:

$$
M \dot{s}_{q}+C s_{q}=-Y_{\mathrm{d} r} \tilde{\theta}_{d}+\hat{Y}_{\mathrm{d} r} \tilde{\theta}_{\mathrm{d} k}-\hat{J}^{T} K_{m} \hat{s}_{x}-K_{p} s_{q}-\left(\Lambda+\tau_{d}\right)
$$

To analyze the stability and convergence of the closedloop system, a Lyapunov candidate function is given as:

$$
\begin{aligned}
V= & \frac{1}{2}\left(s_{q}^{T} M s_{q}+\tilde{\theta}_{d}^{T} P_{d}^{-1} \tilde{\theta}_{d}+\tilde{\theta}_{\mathrm{d} k}^{T} L_{\mathrm{d} k}^{-1} \tilde{\theta}_{\mathrm{d} k}\right. \\
& \left.+\tilde{\theta}_{k}^{T} P_{k}^{-1} \tilde{\theta}_{k}+\sum_{i=1}^{n} \tilde{D}_{i}^{T} L_{r}^{-1} \tilde{D}_{i}\right)+\alpha \tilde{x}^{T} K_{m} \tilde{x}
\end{aligned}
$$

Differentiating $V$ with respect to time leads to:

$$
\begin{aligned}
\dot{V}= & s_{q}^{T}\left(M \dot{s}_{q}+\frac{1}{2} \dot{M} s_{q}\right)+\tilde{\theta}_{d}^{T} P_{d}^{-1} \dot{\hat{\theta}}_{d}+\frac{1}{2} \tilde{\theta}_{d}^{T} \dot{P}_{d}^{-1} \tilde{\theta}_{d}+\tilde{\theta}_{\mathrm{d} k}^{T} L_{\mathrm{d} k}^{-1} \dot{\hat{\theta}}_{\mathrm{d} k} \\
& +\sum_{i=1}^{n} \tilde{D}_{i}^{T} L_{r}^{-1} \dot{\hat{D}}_{i}+\frac{1}{2} \tilde{\theta}_{\mathrm{d} k}^{T} \dot{L}_{\mathrm{d} k}^{-1} \tilde{\theta}_{\mathrm{d} k}+\tilde{\theta}_{k}^{T} P_{k}^{-1} \dot{\hat{\theta}}_{k} \\
& +\frac{1}{2} \tilde{\theta}_{k}^{T} \dot{P}_{k}^{-1} \tilde{\theta}_{k}+2 \alpha \tilde{x}^{T} K_{m} \dot{\tilde{x}}
\end{aligned}
$$

Using the skew-property of the matrix $\dot{M}(q)-2 C(q, \dot{q})$ and substituting (36-39), (40), (42) and (46) into (48) yields:
According to (35) and Lemma 3, the following two inequalities can be obtained, respectively:

$$
-s_{q i} \tau_{d} \leq\left|s_{q i}\right| D_{i}
$$

$$
-\tilde{D}_{i}^{T} L_{r}^{-1} \aleph \hat{D}_{i} \leq-\frac{1}{2} \tilde{D}_{i}^{T} L_{r}^{-1} \aleph \tilde{D}_{i}+\frac{1}{2} D_{i}^{T} L_{r}^{-1} \aleph D_{i}
$$

Substituting the above inequalities with (34) into $\dot{V}_{2}$ and considering Lemma 1 yields:

$$
\begin{aligned}
\dot{V}_{2}= & -\sum_{i=1}^{n} s_{q i}\left(\Lambda_{i}+\tau_{d i}\right)+\sum_{i=1}^{n}\left(\tilde{D}_{i}^{T} s_{q i} \tanh \left(s_{q i} / \varsigma\right)\right. \\
& \left.-\tilde{D}_{i}^{T} L_{r}^{-1} \aleph \hat{D}_{i}\right) \\
\leq & \sum_{i=1}^{n}\left\{D_{i}\left|s_{q i}\right|+\left(\tilde{D}_{i}-\hat{D}_{i}\right) s_{q i} \tanh \left(s_{q i} / \varsigma\right)\right. \\
& \left.-\frac{1}{2} \tilde{D}_{i}^{T} L_{r}^{-1} \aleph \tilde{D}_{i}+\frac{1}{2} D_{i}^{T} L_{r}^{-1} \aleph D_{i}\right\} \\
\leq & \sum_{i=1}^{n}\left(\delta \varsigma D_{i}-\frac{1}{2} \tilde{D}_{i}^{T} L_{r}^{-1} \aleph \tilde{D}_{i}+\frac{1}{2} D_{i}^{T} L_{r}^{-1} \aleph D_{i}\right)
\end{aligned}
$$

Furthermore, according to the definition of $\Delta \hat{\dot{x}}, \dot{\tilde{x}}$ and $\tilde{\theta}_{k}$, we obtain: 


$$
\Delta \hat{\dot{x}}-\dot{\tilde{x}}+Y_{k} \tilde{\theta}_{k}=0
$$

With (24) and (25), we have:

$$
\begin{aligned}
\left(\hat{J} s_{q}\right)^{T} K_{m} \hat{s}_{x} & =\hat{s}_{x}^{T} K_{m} \hat{s}_{x} \\
& =(\Delta \hat{\dot{x}}+\alpha \tilde{x})^{T} K_{m}(\Delta \hat{\dot{x}}+\alpha \tilde{x}) \\
& =\Delta \hat{\dot{x}}^{T} K_{m} \Delta \hat{\dot{x}}+2 \alpha \Delta \hat{\dot{x}}^{T} K_{m} \tilde{x}+\alpha^{2} \tilde{x}^{T} K_{m} \tilde{x}
\end{aligned}
$$

Substituting (53) and (54) into $\dot{V}_{3}$ leads to:

$$
\dot{V}_{3}=-\Delta \hat{\dot{x}}^{T} K_{m} \Delta \hat{\dot{x}}-\alpha^{2} \tilde{x}^{T} K_{m} \tilde{x}
$$

Similar to (51), $\dot{V}_{4}$ can be reformulated as:

$$
\dot{V}_{4} \leq-\frac{1}{2} \tilde{\theta}_{\mathrm{d} k}^{T} L_{\mathrm{d} k}^{-1} \Im \tilde{\theta}_{\mathrm{d} k}+\frac{1}{2} \theta_{\mathrm{d} k}^{T} L_{\mathrm{d} k}^{-1} \Im \theta_{\mathrm{d} k}
$$

Considering that $\lambda_{d}$ and $\lambda_{k}$ are uniformly non-negative, then Equation (49) can be reformulated as follows by substituting (52), (55) and (56) in it,

$$
\begin{aligned}
\dot{V} \leq & -s_{q}^{T} K_{p} s_{q}-\frac{\lambda_{d}}{2} \tilde{\theta}_{d}^{T} P_{d}^{-1} \tilde{\theta}_{d}-\frac{1}{2} \tilde{\theta}_{d}^{T} W_{d}^{T} R_{d} W_{d} \tilde{\theta}_{d} \\
& -\frac{\lambda_{k}}{2} \tilde{\theta}_{k}^{T} P_{k}^{-1} \tilde{\theta}_{k}-\frac{1}{2} \tilde{\theta}_{k}^{T} W_{k}^{T} R_{k} W_{k} \tilde{\theta}_{k} \\
& -\Delta \hat{\dot{x}}^{T} K_{m} \Delta \hat{\dot{x}}-\alpha^{2} \tilde{x}^{T} K_{m} \tilde{x} \\
& +\sum_{i=1}^{n}\left(\delta \varsigma D_{i}-\frac{1}{2} \tilde{D}_{i}^{T} L_{r}^{-1} \aleph \tilde{D}_{i}+\frac{1}{2} D_{i}^{T} L_{r}^{-1} \aleph D_{i}\right) \\
& -\frac{1}{2} \tilde{\theta}_{\mathrm{d} k}^{T} L_{\mathrm{d} k}^{-1} \Im \tilde{\theta}_{\mathrm{d} k}+\frac{1}{2} \theta_{\mathrm{d} k}^{T} L_{\mathrm{d} k}^{-1} \Im \theta_{\mathrm{d} k}
\end{aligned}
$$

Define a positive constant $\eta$ as:

$$
\eta=\min \left(\frac{2 \omega_{\min }\left(K_{p}\right)}{\omega_{\max }(M(q))}, \lambda_{d}, \lambda_{k}, \Im, \aleph, \alpha\right)
$$

where $\omega_{\min }(\cdot)$ and $\omega_{\max }(\cdot)$ denote the minimum and maximum eigenvalues of the related matrix respectively. Then (57) can be rewritten as:

$$
\dot{V} \leq-\eta V+v
$$

where $\quad v=\frac{1}{2} \theta_{\mathrm{d} k}^{T} L_{\mathrm{d} k}^{-1} \Im \theta_{\mathrm{d} k}+\sum_{i=1}^{n}\left(\delta \varsigma D_{i}+\frac{1}{2} D_{i}^{T} L_{r}^{-1} \aleph D_{i}\right)$. According to (59), a conclusion that the Lyapunov function $V$ is non-increasing can be obtained.

Now we are in the position to state the following theorem:

Theorem 1: Under the assumption that two estimated task Jacobian $\hat{J}_{1}$ and $\hat{J}_{2}$ are uniformly independent, then the adaptive control law (33) with parameter update laws (36-39) can guarantee the stability of control system for redundant manipulators and lead to the convergence of the tracking errors of the two prioritized tasks. That is $x_{1}-x_{1 d} \rightarrow 0, x_{2}-x_{2 d} \rightarrow 0$ and $\dot{x}_{1}-\dot{x}_{1 d} \rightarrow 0, \dot{x}_{2}-\dot{x}_{2 d} \rightarrow 0$ as $t \rightarrow 0$.

Proof: Please see Appendix 1.
Remark 3: If $W_{d}$ and $W_{k}$ are both persistent exciting, $\lambda_{d}>0$ and $\lambda_{k}>0$ always hold. This results in the fact that $\tilde{\theta}_{d} \in \mathcal{L}_{2} \cap \mathcal{L}_{\infty}$ and $\tilde{\theta}_{k} \in \mathcal{L}_{2} \cap \mathcal{L}_{\infty}$ when considering (57). In addition, $\tilde{\tilde{\theta}}_{d}$ and $\tilde{\theta}_{k}$ are bounded since $\dot{\hat{\theta}}_{d}$ and $\dot{\hat{\theta}}_{k}$ in (36) and (38) are bounded. Then $\hat{\theta}_{d}-\theta_{d} \rightarrow 0$ and $\hat{\theta}_{k}-\theta_{k} \rightarrow 0$ can be achieved.

Remark 4: The stability and convergence analysis above suffers from four kinds of singularities: kinematic singularities of the task Jacobian and that of its estimated ones, algorithmic singularities of the projected Jacobian and that of its estimated one. Before handling them, their characteristics should be discussed separately for differential treatment. For two given prioritized tasks, the kinematic singularities of $J_{1}$ and $J_{2}$ are determined in advance and will not be considered when assuming that the redundant manipulator is operating in two finite task spaces. In addition, a standard projection algorithm can be used to ensure that the estimated kinematic parameter $\hat{\theta}_{k}$ remains in an appropriate region [30]. Therefore, kinematic singularities of the estimated task Jacobian $\hat{J}_{1}\left(q, \theta_{k}\right)$ and $\hat{J}_{2}\left(q, \theta_{k}\right)$ only depend on $q$, not $\hat{\theta}_{k}$, which further results in the fact the full rank of the two estimated task Jacobian can always holds during the adaptation.

From (21), rank deficiency of $\hat{\bar{J}}_{2}$ leads to the ill-conditioned solution of $q_{r}$. So only the algorithmic singularity of $\bar{J}_{2}$ and $\hat{\bar{J}}_{2}$, will be discussed in detail in the next part.

\subsection{Dependent case}

For sake of simplicity, algorithmic singularities are avoided in the above derivation by several proper assumptions. This will make the controller to loss generality as to dependent case. The algorithmic singularities of $\bar{J}_{2}$ with $\hat{\bar{J}}_{2}$ are difficult to predict since they depend on how the two tasks conflict with each other along the desired trajectory being executed. So, we will propose a modified adaptive controller to achieve the main goal of task tracking while release these restrictive assumptions simultaneously.

A singularity-robust inverse (SRI) method from the field of inverse kinematics, which is known as damped least square technique (DLS), can be used here to cope with the algorithmic singularities. The most general approach concerning DLS is based upon variable damping factors [31]. Although this approach can ensure continuity and good shaping of DLS solution by choosing the damping factor $\gamma$ as (48), it is not intuitive as pointed in Ref. [32] and the direct consequence on physical values of the system is not clear, because it creates unnecessary damping for other well-conditioned singular vector, this may further produce more tracking error.

$$
\gamma^{2}=\left\{\begin{array}{l}
0 \quad\left(\sigma_{m_{2}} \geq \varepsilon\right) \\
\left(1-\left(\frac{\sigma_{m_{2}}}{\varepsilon}\right)^{2}\right) \gamma_{\max }^{2} \quad\left(\sigma_{m_{2}}<\varepsilon\right)
\end{array}\right.
$$


Evoked by the SRI approach proposed in Ref. [33], Gaussian distribution of damping factor can be adopted here and only the ill-conditioned singular vectors are damped by applying singular value decomposition (SVD) [34]. Then (21) should be reformulated as:

$$
\begin{gathered}
\dot{q}_{r}=\hat{J}_{1}^{\dagger} \dot{x}_{1 r}+\hat{N}_{1}\left(\hat{\bar{J}}_{2}\right)^{*}\left(\dot{x}_{2 r}-\hat{J}_{2} \hat{J}_{1}^{\dagger} \dot{x}_{1 r}\right) \\
\left(\hat{\bar{J}}_{2}\right)^{*}=\sum_{i=1}^{m_{2}} \frac{\sigma_{i}}{\sigma_{i}^{2}+\lambda_{G i}^{2}} v_{i} \mu_{i}^{T} \\
\lambda_{G i}=\lambda_{\max } \exp \left(-\left(\sigma_{i} / \varepsilon\right)^{2}\right)
\end{gathered}
$$

where $\sigma_{i}$ is the singular value of the estimated projected Jacobian $\hat{\bar{J}}_{2}, v_{i}$ and $\mu_{i}$ are the $i$-th output and input singular vector respectively. $\varepsilon$ is the pre-specified constant which defines the size of the singular region, $\lambda_{\max }$ sets the maximum of the damping factor. Optimal choice of aforementioned two parameters should be obtained by trial and error.

When approaching to algorithmic singularities, nonnull damping factor brings in inevitable reconstruction error to the subtask tracking, which will further lead to the unpredictable parameter estimation and torque discontinuity. Significantly, discontinuous command torques would result in unstable behavior. Hence, the control law and kinematic parameter updating law in (33) and (38) should also be properly modified. The idea of continuous dead zone [29] and transition shaping technique [32] can be used here to avoid unexpected parameter adaptation and discontinuity of the control torques brought by the reconstruction tracking error of the subtask.

$$
\tau=Y_{\mathrm{d} r} \hat{\theta}_{d}-\hat{Y}_{\mathrm{d} r} \hat{\theta}_{d 1}+\hat{J}_{1}^{T} K_{m 1} \hat{s}_{x 1}+\mu \hat{J}_{2}^{T} K_{m 2} \hat{s}_{x 2}+K_{p} s_{q}+\Lambda
$$

$$
\dot{\hat{\theta}}_{k}=P_{k}(t)\left[-2 \alpha\left(Y_{k 1}^{T} K_{m 1} \tilde{x}_{1}+\mu Y_{k 2}^{T} K_{m 2} \tilde{x}_{2}\right)-W_{k}^{T} R_{k} e_{k}\right]
$$

$$
\mu=\left\{\begin{array}{l}
1 \quad\left(\text { for } i=1, \ldots, m \lambda_{G i} \leq \Delta\right) \\
\exp \left(-\left(1-\lambda_{G i} / \Delta\right)^{2}\right) \quad\left(\lambda_{G i}>\Delta\right)
\end{array}\right.
$$

where $\Delta$ is a small positive preassigned threshold. From remark $1, \mu$ is introduced to determine whether or not the adaptation and control term of tracking error of the subtask are activated for parameter estimation and control laws.

The stability and convergence proof for primary task when choosing (64), (36), (37), (39) and (65) as the control law and updating laws are similar to that of Theorem 1.

$$
\begin{aligned}
V_{p}= & \frac{1}{2}\left(s_{q}^{T} M s_{q}+\tilde{\theta}_{\mathrm{d} k}^{T} L_{\mathrm{d} k}^{-1} \tilde{\theta}_{\mathrm{d} k}+\tilde{\theta}_{d}^{T} P_{d}^{-1} \tilde{\theta}_{d}+\tilde{\theta}_{k 1}^{T} P_{k}^{-1} \tilde{\theta}_{k 1}\right) \\
& +\alpha \tilde{x}_{1}^{T} K_{m} \tilde{x}_{1}
\end{aligned}
$$

An alternative approach is to use the following redundancy resolution equation to replace (21)

$$
\dot{q}_{r}=\hat{J}_{1}^{\dagger} \dot{x}_{1 r}+\hat{N}_{1} \hat{J}_{2}^{\dagger} \dot{x}_{2 r}
$$

In which the algorithmic singularities are avoided in the cost of a greater tracking error of the subtask [35].

Remark 5: When we preassign the prioritized multiple tasks, the basic principle is to ensure that they are not uniformly dependent, hopefully they are uniformly independent (otherwise the subtasks are meaningless and loss the possibility to be well executed). So, the algorithmic singularity is usually temporary. As stated in Remark 1, the tracking error and prediction error of the subtask then have much space to be utilized for faster convergence rate and better tracking accuracy of the multiple tasks. When algorithmic singularity is encountered, the modified scheme in this section can properly avoid the unexpected parameter adaptation and unpredictable commanded torques.

Remark 6: Fast resolution of the inverse can resort to a hierarchized complete orthogonal decomposition method [36].

\section{Simulation results}

In this section, we present the simulation results of a threeDOF planar manipulator grasping an unknown welding pistol to verify the performance of the robust adaptation based multi-priority controller proposed above (RAM Scheme for short). The simulation is implemented by Simulink and SimMechanics. The block diagram is shown in Figure 2.

Since there is no controller with the same aim in the literatures (to the author's best knowledge), to demonstrate the advantages of the proposed RAM in the robustness, tracking performance and the smoothness of the commanded torques, another controller is established for comparison, which is a direct combination of the adaptive Jacobian controller proposed by Cheah in Ref. [12] and the priority oriented adaptive controller proposed by $\mathrm{Dr}$ Sedeghian in Ref. [19], and is abbreviated as C-H scheme hereinafter. Common parameters of the two comparative schemes share same values.

We assume that the position of the end-point of the tool can be gained from the robot vision system. Physical parameters of the system are listed in Table $1 . m_{1}, m_{2}, m_{3}$ and $m_{e}$ are the masses of the three links and the tool; $I_{i}$ for 


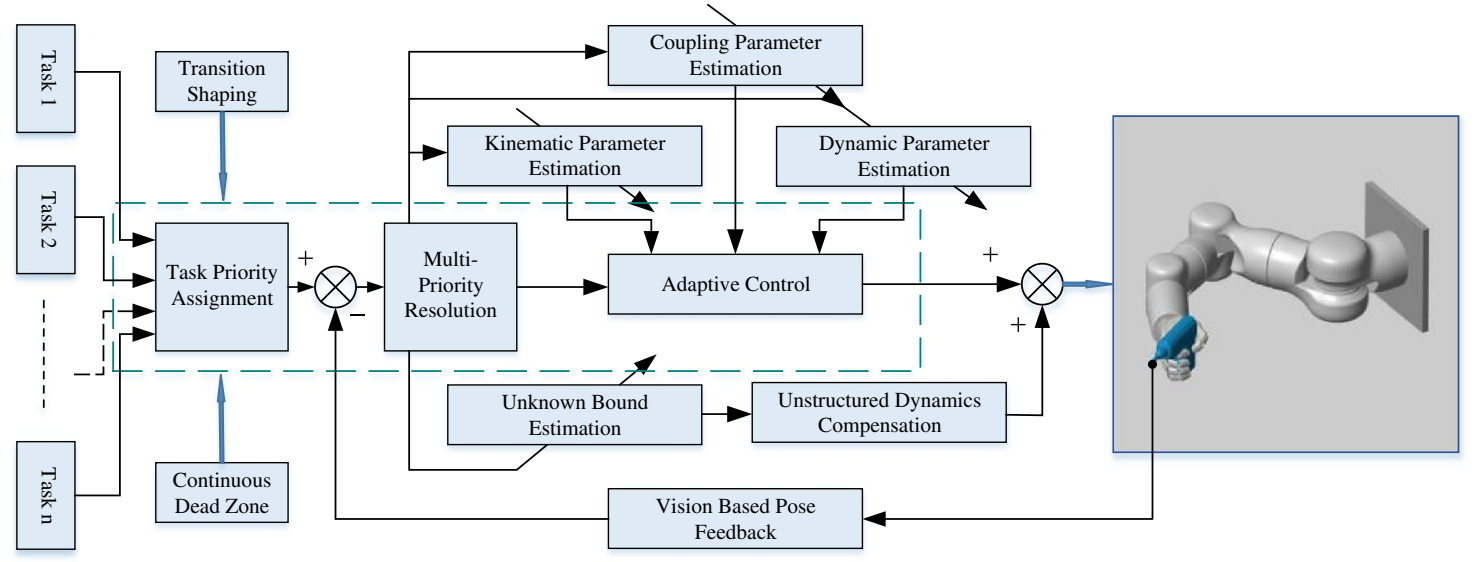

Figure 2. Block diagram of the proposed controller for redundant manipulator with unknown welding pistol.

Table 1. Parameters of the manipulator and tool.

\begin{tabular}{lccll}
\hline Body & $m_{i}(\mathrm{~kg})$ & $l_{i}\left(\mathrm{~kg} \cdot \mathrm{m}^{2}\right)$ & $l_{i}(\mathrm{~m})$ & $l_{c i}(\mathrm{~m})$ \\
\hline Link (1) & 6.5 & 0.12 & 0.42 & 0.28 \\
Link (2) & 5.0 & 0.42 & 0.3 & 0.22 \\
Link (3) & 2.6 & 0.10 & 0.24 & 0.15 \\
Tool (e) & 1.0 & 0.06 & 0.2 & 0.1 \\
\hline
\end{tabular}

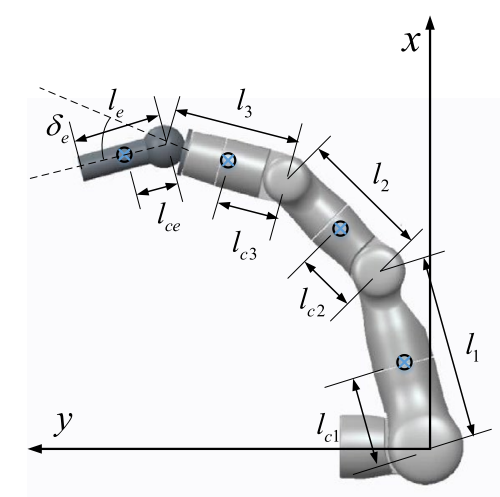

Figure 3. Sketch of the HIT-Robonaut system with an unknown tool.

$(i=1,2,3, e)$ denotes their momentum of inertial. $l_{i}$ and $l_{c i}$, which are shown in Figure 3, express their link length and the centers of mass respectively. The deflection angle is specified as $\delta_{e}=\pi / 6$. One can refer to Appendix 2 for the details about the parameterized linearization of the dynamics and kinematics.

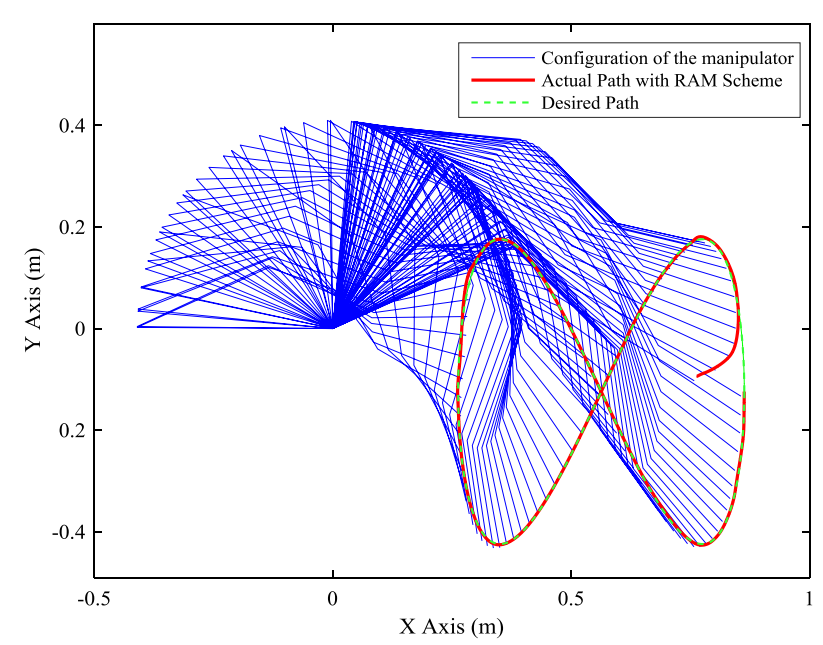

Figure 4. Arm configuration of the 3-DOF planar redundant manipulators.

The initial configuration of the 3-DOF manipulator is $q(0)=[\pi / 3,-\pi / 2,-\pi / 6]^{T}$ and the initial value of the end-effector position is $x_{1}(0)=[0.7630,-0.0941]^{T}$.

In the simulation, the desired end-effector trajectory of the primary task is depicted by: $x_{d 1}=[0.4730+0.3 \cos (0.2 \pi t) \quad-0.1241+0.3 \sin (0.4 \pi t)]^{T}$. The subtask is defined as the square of the length from joint 2 to the end tip. The desired value for this task is given as $x_{2 d}=0.5054$ and the initial value is $x_{2}(0)=0.5154$.

Without loss of generality, the unmodeled error $\tau_{d}$ is consisted of unstructured friction ${ }^{u} \tau_{f}(\dot{q})$ and time-varying term $\tau_{\varsigma}(t)$, where

$$
\left\{\begin{array}{l}
{ }^{u} \tau_{f}(\dot{q})=\operatorname{diag}(0.75,0.3,0.075) \times \dot{q}+\operatorname{diag}(0.1,0.1,0.025) \times \operatorname{sgn}(\dot{q}) \\
\tau_{\varsigma}(t)=[0.5+5 \sin (2 \pi t) ; 0.5+0.25 \sin (2 \pi t) ;-0.5-0.25 \sin (2 \pi t)]
\end{array}\right.
$$


The control parameters of the proposed controller are chosen as:

$$
\begin{gathered}
\lambda_{f}=2, \lambda_{f k 1}=\lambda_{f k 2}=20, \alpha=10, K_{p}=20 I_{3}, \\
\Delta=2 \times 10^{-3}, \aleph=0.1, \mathfrak{\Im}=0.1, \lambda_{k}=5, \\
P_{k}(0)=1.5 I_{6}, R_{k}=0.8 I_{3}, K_{m 1}=0.5 I_{2}, K_{m 2}=0.1 I_{1}, \\
P_{d}(0)=2.5 I_{7}, \lambda_{d}=1, L_{\mathrm{d} k}=1.2, \\
R_{d}=5 I_{3}, \hat{\theta}_{k}(0)=[0.6,0.5,0.2,0.05,0.2,0.1]^{T}, \\
\theta_{k}=[0.42,0.3,0.4132,0.1,0.124,0.03]^{T}, \\
\hat{\theta}_{d}(0)=50 \% \times \theta_{d}, \\
\theta_{d}=[3.4603,1.3137,0.3277,0.462,0.3017,0.4536,0.2155]^{T} .
\end{gathered}
$$

The two parameters of the Gaussian-based DLS method are chosen as: $\lambda_{\max }=0.1, \varepsilon=0.04$. The parameters of the robust compensator are chosen as: $\varsigma=0.01, L_{r}=1$.

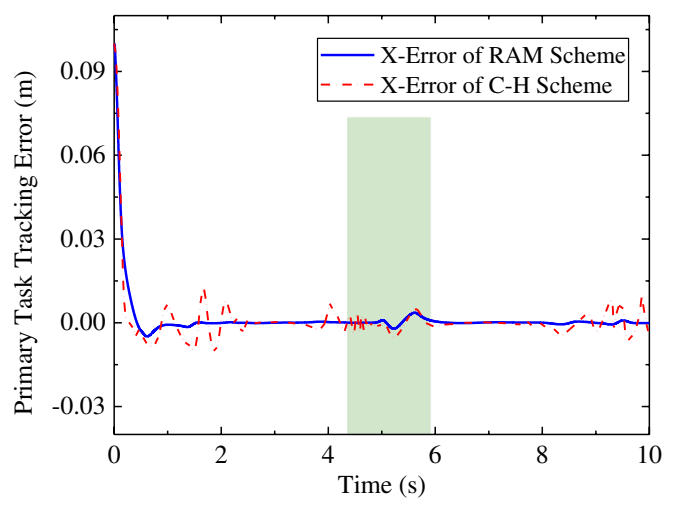

a) Primary task tracking errors withtwo schemes in $\mathrm{X}$-axis

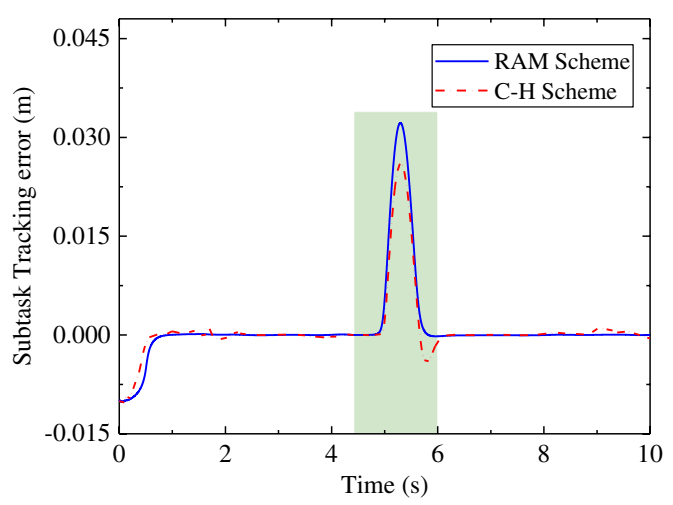

c) Subtask tracking errorswith two schemes

\subsection{Performance analysis of the RAM scheme}

With 50\% dynamic uncertainty and nearly $40 \%$ kinematic uncertainty and the unstructured disturbance described by (69), the corresponding simulation results are illustrated in Figures 4-7. The arm configuration trajectory with RAM scheme is presented in Figure 4, from which we can see that the primary task is well tracked.

From these figures, we can obtain the following remarks about the proposed RAM scheme:

(1) Even the parameter adaptation process is in company with algorithmic singularity at the beginning ( $0 \mathrm{~s} \leq t<0.6 \mathrm{~s})$, the convergence of the tracking error of the two tasks are not disturbed (show by the blues solid line in Figure $5(\mathrm{a})-(\mathrm{c}))$.

(2) Within the time interval of shaded rectangle (from $t=4.5 \mathrm{~s}$ to $t=6 \mathrm{~s}$ ) in Figures 5 (a)-(c) and $6(\mathrm{a})$, the performance of the proposed RAM scheme near algorithmic singularity is

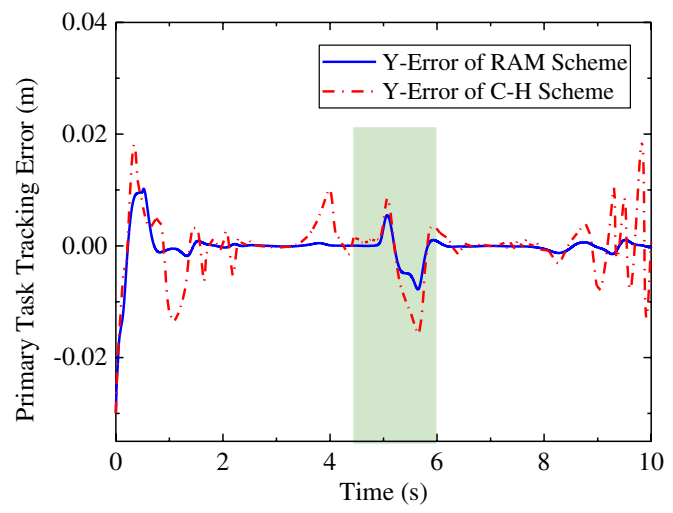

b) Primary task tracking errors withtwo schemes in $\mathrm{Y}$-axis

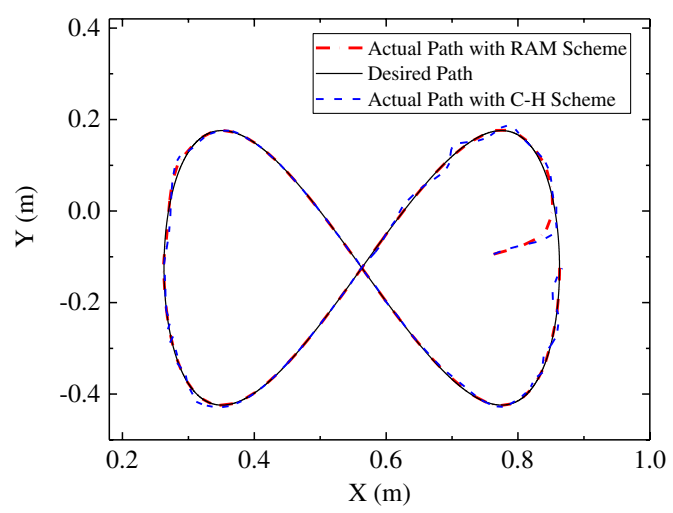

d) Primary tracking results withtwo schemes in X-Y Panal

Figure 5. Multi-task tracking results with two comparative schemes. (a) Primary task tracking errors with two schemes in $X$-axis, (b) Primary task tracking errors with two schemes in $Y$-axis, (c) Subtask tracking errors with two schemes and (d) Primary tracking results with two schemes in $X$ - $Y$ Panal. 


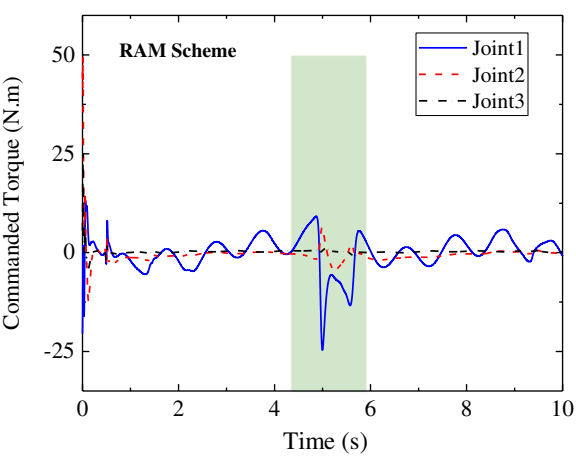

a) Joint commanded torques with RAM scheme

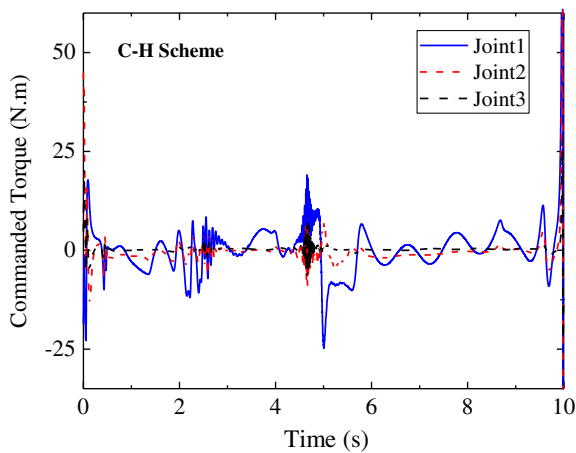

b) Joint commanded torques with $\mathrm{C}-\mathrm{H}$ scheme

Figure 6. Joint commanded torques with two comparative schemes. (a) Joint commanded torques with RAM scheme and (b) Joint commanded torques with $\mathrm{C}-\mathrm{H}$ scheme.

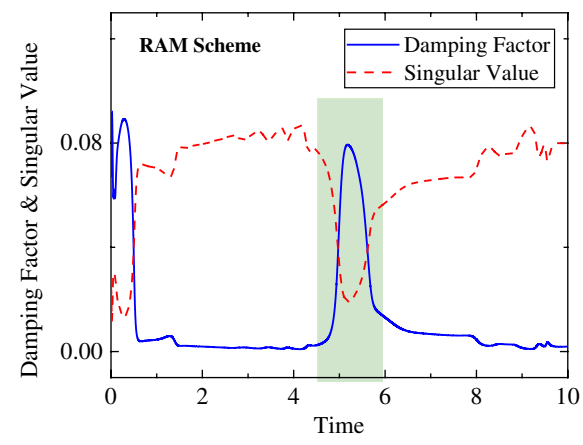

a) The damping factor and singular value of the projected Jacobian

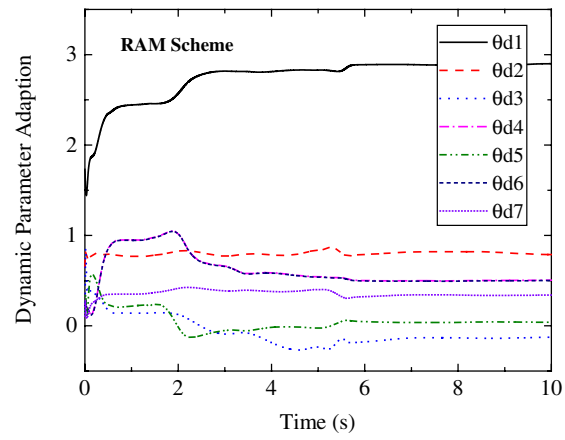

c) The estimates of the Dynamicparameters

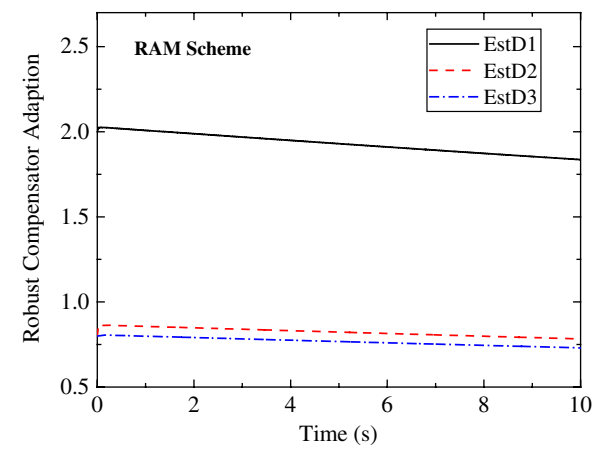

b) The estimates of the bounds

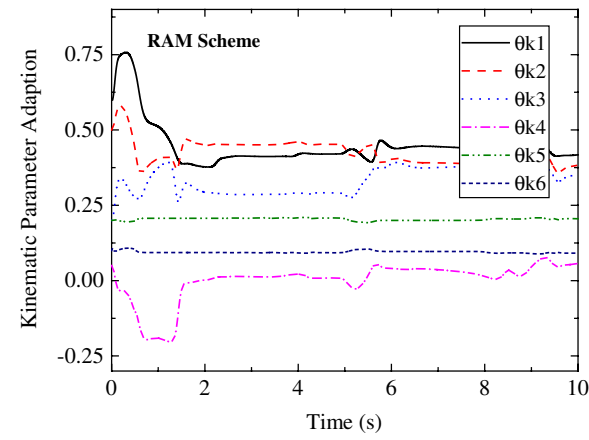

d) The estimates of the kinematic parameters

Figure 7. Parameter adaption results with the proposed RAM scheme. (a) The damping factor and singular value of the projected Jacobian, (b) The estimates of the bounds, (c) The estimates of the Dynamic parameters and (d) The estimates of the kinematic parameters.

presented. The commanded torques are kept continuous and the tracking performance of the subtask is sacrificed to retain high precision of the primary task. For clear understanding of the task dependence condition during the simulation process, one can refer to Figure $7(\mathrm{a})$ which shows the singular value of the estimated projected Jacobian and the corresponding damping factor (calculated by the improved SRI).

(3) When far from the region of algorithmic singularity $(1 \mathrm{~s} \leq t \leq 4.5 \mathrm{~s}$ and $6 \mathrm{~s}<t \leq 10 \mathrm{~s})$, both of the two tasks are performed well.

(4) Even with small adaptation and control gains, tracking error of the primary task still converges quickly. 


\subsection{Comparison results analysis}

Figure 5 shows the comparison results of the multi-task tracking performance between the RAM scheme and the combined $\mathrm{C}-\mathrm{H}$ scheme. From the four panels, we see that faster convergence rate and smaller tracking error in both primary task and subtask can be achieved by the proposed RAM when facing dynamic and kinematic uncertainties and unstructured disturbances. This is because the proposed RAM properly utilizes both the prediction error and tracking error of the primary task and subtask to accelerate the adaptation process, and the unstructured disturbances are suppressed by an adaptive robust compensator.

Furthermore, Figure 6(a) and (b) present the joint commanded torques with RAM scheme and the combined $\mathrm{C}-\mathrm{H}$ scheme, respectively. From the two panels, we see that the commanded torques with RAM always maintain continuous and smooth and little chattering exists even with a robust compensator. This superiority owes to the combined action of continuous differentiable switch function of the robust compensator, the continuous dead zone and torque transition shaping. These incorporated techniques guarantee the easy application in along with improvement of the tracking performance.

\section{Conclusion and discussion}

In this paper, a novel robust adaptive multi-priority controller is proposed to solve the multi-task tracking problem of the redundant manipulators when dynamic/kinematic uncertainties and unstructured dynamics exist. To accelerate the adaptation process, both the tracking errors of the two prioritized tasks and the prediction error are utilized. Moreover, a continuous robust compensator is designed to suppress the unstructured disturbances. We discuss and design the controller in the sense of algorithmic singularity. Unlike the general damping least square method, an improved singularity-robust technique is incorporated to minimize the effect the damping factor acting on the task tracking and alleviate the bad behaviour of damped projected Jacobian. The idea of continuous dead zone is used to cope with the problem of unpredictable adaptation when reconstruction error exists in the subtask. Besides, transition shaping technique is also applied to the commanded torques to eliminate the discontinuity led by the potential discontinuous projection. Along with the improvement of the multi-task tracking performance, smoothness of the commanded torques is still guaranteed and the noisy joint accelerations/task velocities are not required. Simulation results have verified the effectiveness and universality of the proposed controller whether facing algorithmic singularity or not.

It is worth mentioning that two factors may affect the practical performance of the proposed controller when implementing the corresponding experiments: sampling time and measurement error of the vision system. By improving the visual algorithm of object detection/ tracking and stereo matching, reduction of image processing time can be expected. Furthermore, Kalman filters or extended Kalman filters can be used and properly designed for removal of measurement error based on characteristic analysis.

\section{Acknowledgment}

The authors would like to express their great appreciation to the anonymous reviewers for their precious time. Special thanks to Dr. Hanlei Wang and Dr. Zhengsheng Chen for their suggestions and Dr Baoshi Cao for the provision of robot illustration.

\section{Disclosure statement}

No potential conflict of interest was reported by the authors.

\section{Notes on contributors}

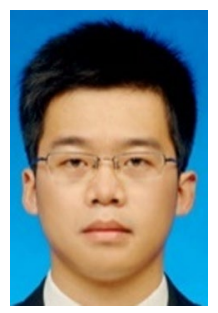

Yi Ren is currently a $\mathrm{PhD}$ candidate in State Key Laboratory of Robotics and System at Harbin Institute of Technology (HIT) in China. He received his BS (2011) in Thermal Energy and Power Engineering and MS (2013) in Mechatronics Engineering at HIT. His research interests include robotics, control of redundant manipulator/dual-arm cooperation, and object grasping.

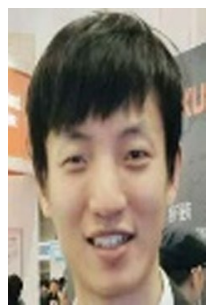

Yang Zhou received his MS (2008) in Electronic Science and Technology and PhD (2014) in Mechatronics Engineering from Harbin Institute of Technology (HIT), Harbin, PRC. Now he is a researcher in Beijing Institute of Control Engineering (BICE). His research interests focus on the robot control system, dextrous manipulation, and control of dual-arm systems.

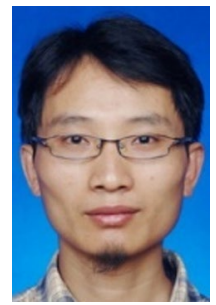

Yechao Liu received his BS (2002) in Thermal Energy and Power Engineering and MS (2004), PhD degrees (2009) in Mechatronics Engineering from Harbin Institute of Technology (HIT), Harbin, PRC. Now he is an associate professor at the school of Mechanical Engineering in HIT. His research interests are in the area of robotics, dynamics algorithms of flexible-joint manipulators, and their compliance control.

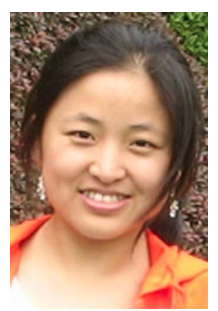

Yikun $\mathrm{G} u$ received her $\mathrm{MS}$ and $\mathrm{PhD}$ degrees in Mechatronics Engineering from Harbin Institute of Technology, Harbin, China, in 2006 and 2011, respectively. She is now in The Johns Hopkins University as a visiting scholar. Her research interests include ac motor drives, nonlinear adaptive control, neural networks, and flexible joint robot arm control. 


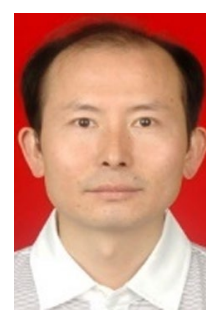

Minghe Jin received his BS degree in Vehicle Engineering in 1993, and the MS and PhD degrees in Mechanical Engineering, in 1996 and 2000, respectively, all from Harbin Institute of Technology (HIT), Harbin, PRC. Now he is professor in Robotics Institute in HIT. His research interests include dexterous robot hands, space robotics, and real-time embedded control system.

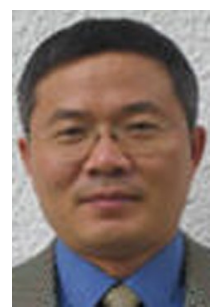

Hong Liu received the $\mathrm{PhD}$ degree in 1993 from Harbin Institute of Technology, PRC. During 1991-1993 he worked as a Joint-PhD candidate in the Institute of Robotics and System Dynamics at the German Aerospace Research Establishment (DLR) in Germany. Since 1993 he has been a Research Fellow in this institute. He became one of the first batch of Changjiang Scholars in 1998. He is currently a professor in HIT. His research projects include the development of a dexterous robot hand, space manipulator, etc.

\section{Funding}

This work was supported by the National Nature Science Foundation of China [grant number 61603112].

\section{References}

[1] Borst C, Wimböck T, Schmidt F, et al. Rollin'Justin-mobile platform with variable base. In 2009 IEEE International Conference on Robotics and Automation; 2009 May 1217; Kobe, Japan; 2009. p. 1597-1598.

[2] Diftler, MA, Mehling JS, Abdallah ME, et al. Robonaut 2-the first humanoid robot in space. In 2011 IEEE International Conference on Robotics and Automation (ICRA). IEEE; 2011 May 9-13; Shanghai, China; 2011. p. $2178-2183$.

[3] Kaloust J, Zhihua Q, Ham C. Nonlinear robust control design for robot manipulators with unmodeled actuator dynamics. Adv Rob. 1995;10(5):453-467.

[4] Patel RV, Talebi HA, Jayender J, et al. A robust position and force control strategy for 7-DOF redundant manipulators. IEEE/ASME Trans Mechatron. 2009;14(5):575-589.

[5] Middleton RH, Goodwin GC. Adaptive computed torque control for rigid link manipulations. Syst Control Lett. 1988;10(1):9-16.

[6] Li W, Slotine J-JE. An indirect adaptive robot controller. Syst Control Lett. 1989;12(3):259-266.

[7] Slotine J-JE, Li W. Composite adaptive control of robot manipulators. Automatica. 1989;25(4):509-519.

[8] Feng G. A new adaptive control algorithm for robot manipulators in task space. IEEE Trans Rob Autom. 1995;11(3):457-462.

[9] Ciliz MK. Combined direct and indirect adaptive control of robot manipulators using multiple models. Adv Rob. 2006;20(4):483-497.

[10] Slotine J-JE, Li W. Applied nonlinear control. Vol. 199(1). Englewood Cliffs, NJ: Prentice-Hall, 1991.

[11] Cheah C-C, Liu C, Slotine JJJE. Adaptive tracking control for robots with unknown kinematic and dynamic properties. Int J Rob Res. 2006;25(3):283-296.
[12] Cheah C-C, Liu C, Slotine JJJE. Adaptive Jacobian tracking control of robots with uncertainties in kinematic, dynamic and actuator models. IEEE Trans Autom Control. 2006;51(6):1024-1029.

[13] Liu C, Cheah CC, Slotine J-JE. Adaptive Jacobian tracking control of rigid-link electrically driven robots based on visual task-space information. Automatica. 2006;42(9):1491-1501.

[14] Galicki M. Inverse-free control of a robotic manipulator in a task space. Rob Autonom Syst. 2014;62(2):131-141.

[15] Wang H, Xie Y. Adaptive inverse dynamics control of robots with uncertain kinematics and dynamics. Automatica. 2009;45(9):2114-2119.

[16] Wang H, Xie Y. Prediction error based adaptive jacobian tracking of robots with uncertain kinematics and dynamics. IEEE Trans Autom Control. 2009;54(12):28892894.

[17] Choi Y, Oh Y, Oh SR, et al. Multiple tasks manipulation for a robotic manipulator. Adv Rob. 2004;18(6):637-653.

[18] Tatlicioglu E, Braganza D, Burg TC, et al. Adaptive control of redundant robot manipulators with sub-task objectives. Robotica. 2009;27(06):873-881.

[19] Sadeghian H, Keshmiri M, Villani L, et al. Priority oriented adaptive control of kinematically redundant manipulators. In 2012 IEEE International Conference on Robotics and Automation (ICRA). IEEE; 2012 May 14-18; St. Paul, MN; 2012. p. 293-298.

[20] Ott C, Dietrich A, Albu-Schäffer A. Prioritized multitask compliance control of redundant manipulators. Automatica. 2015;53:416-423.

[21] Liu Z, Chen C, Zhang Y. Decentralized robust fuzzy adaptive control of humanoid robot manipulation with unknown actuator backlash. IEEE Trans Fuzzy Syst. 2015;23(3):605-616.

[22] Zhou J, Zhang C, Wen C. Robust adaptive output control of uncertain nonlinear plants with unknown backlash nonlinearity. IEEE Trans Autom Control. 2007;52(3):503-509.

[23] Siciliano B, Sciavicco L, Villani L, et al. Robotics: modelling, planning and control. Berlin: Springer Science \& Business Media; 2010.

[24] Nakamura Y, Hanafusa H, Yoshikawa T. Task-priority based redundancy control of robot manipulators. The International Journal of Robotics Research. 1987;6(2):315.

[25] Siciliano B, Slotine J-JE. A general framework for managing multiple tasks in highly redundant robotic systems. In Fifth International Conference on Advanced Robotics, 1991. 'Robots in Unstructured Environments', 91 ICAR. IEEE; 1991 June 19-22; Ferrara, Italy; 1991. p. 1211-1216.

[26] Antonelli Gianluca. Stability analysis for prioritized closed-loop inverse kinematic algorithms for redundant robotic systems. IEEE Trans Rob. 2009;25(5):985-994.

[27] Ping H, Bodson M, Sastry S, et al. Adaptive identification and control for manipulators without using joint accelerations. In Proceedings of the IEEE International Conference on Robotics and Automation. 1987 Mar 31Apr 3; Raleigh, NC; 1987. p. 1210-1215.

[28] Dietrich A, Ott C, Stramigioli S. Passivation of projectionbased null space compliance control via energy tanks. IEEE Robot Autom Lett. 2016;1(1):184-191. 
[29] Ioannou PA, Sun J. Robust adaptive control. Dover edition. Upper Saddle River, NJ: Courier Corporation; 2012.

[30] Dixon WE. Adaptive regulation of amplitude limited robot manipulators with uncertain kinematics and dynamics. IEEE Trans Autom Control. 2007;52(3):488493.

[31] Nakamura Y, Hanafusa H. Inverse kinematic solutions with singularity robustness for robot manipulator control. J Dyn Syst Meas Contr. 1986;108(3):163-171.

[32] Dietrich A, Wimböck T, Albu-Schäffer A, et al. Integration of reactive, torque-based self-collision avoidance into a task hierarchy. IEEE Trans Rob. 2012;28(6):1278-1293.

[33] Phuoc LM, Martinet P, Lee S, et al. Damped least square based genetic algorithm with Gaussian distribution of damping factor for singularity-robust inverse kinematics. J Mech Sci Technol. 2008;22(7):1330-1338.

[34] Chiaverini S. Singularity-robust task-priority redundancy resolution for real-time kinematic control of robot manipulators. IEEE Trans Rob Autom. 1997;13(3):398410.

[35] Antonelli G, Arrichiello F, Chiaverini S. The null-spacebased behavioral control for autonomous robotic systems. Intel Serv Robot. 2008;1(1):27-39.

[36] Escande A, Mansard N, Wieber P-B. Fast resolution of hierarchized inverse kinematics with inequality constraints. In ICRA 2010-IEEE International Conference on Robotics and Automation; 2010 May 3-8; Anchorage, AL; 2010. p. 3733-3738.

\section{Appendix 1. Proof of Theorem 1}

$\dot{V} \leq-\eta V+v$ implies that $V$ in (47) is always bounded and so as to the integral of $\dot{V}$. This means that $s_{q} \in \mathcal{L}_{\infty} \cap \mathcal{L}_{2}$, $\tilde{x} \in \mathcal{L}_{\infty} \cap \mathcal{L}_{2}, \tilde{\theta}_{k}, \tilde{\theta}_{d}, \tilde{\theta}_{\mathrm{d} k}, \tilde{D}_{i} \in \mathcal{L}_{\infty}, \Delta \hat{\dot{x}} \in \mathcal{L}_{2}, W_{d} \tilde{\theta}_{d} \in \mathcal{L}_{2}$ and $W_{k} \tilde{\theta}_{k} \in \mathcal{L}_{2} . \tilde{x}=x_{d}-x \in \mathcal{L}_{\infty}$ leads to the fact that $x \in \mathcal{L}_{\infty}$ and further $q \in \mathcal{L}_{\infty}$, then $\hat{J}\left(q, \hat{\theta}_{k}\right) \in \mathcal{L}_{\infty}$ holds. From (25), we have $\hat{s}_{x} \in \mathcal{L}_{\infty}$, which implies $\Delta \hat{\dot{x}} \in \mathcal{L}_{\infty}$ and further $\hat{\dot{x}} \in \mathcal{L}_{\infty}$ considering (24). Using (16) and (7), $\dot{q} \in \mathcal{L}_{\infty}$ and then $\dot{x} \in \mathcal{L}_{\infty}$ can be obtained.

Conclusion 1: With the previous analysis, $\dot{\tilde{x}} \in \mathcal{L}_{\infty}$ holds $\tilde{x}=x_{d}-x \rightarrow 0$ is achieved.
Furthermore, $\hat{\vec{x}}_{r}=\ddot{x}_{d}+\alpha(\dot{x}-\hat{\dot{x}}) \in \mathcal{L}_{\infty}$ holds; with the fact that $W_{k}(t) \in \mathcal{L}_{\infty}$ and $e_{k}=W_{k} \tilde{\theta}_{k} \in \mathcal{L}_{\infty}$, we have $\hat{\theta}_{k} \in \mathcal{L}_{\infty}$ considering (38). This leads to $\hat{J}_{1}^{\dagger} \in \mathcal{L}_{\infty}$ and $\dot{\bar{N}} \in \mathcal{L}_{\infty}$ under the assumption that $\hat{J}_{1}$ and $\hat{J}_{2} \hat{N}_{1}$ are both of full rank. Then $\hat{\ddot{q}}_{r} \in \mathcal{L}_{\infty}$ can be obtained by (22). From (33) and (2), the fact that $\tau \rightarrow \mathcal{L}_{\infty}$ with $\tau_{d} \rightarrow \mathcal{L}_{\infty}$ and further $\ddot{q} \rightarrow \mathcal{L}_{\infty}$ holds considering that $\Lambda_{i} \in \mathcal{L}_{\infty}$. And hence $\ddot{x} \in \mathcal{L}_{\infty}$ holds considering the time derivative of (7). Based on $\ddot{\tilde{x}}=\ddot{x}_{d}-\ddot{x}$, then $\ddot{\tilde{x}} \in \mathcal{L}_{\infty}$ holds.

Conclusion 2: Using Lemma 1, $\dot{\tilde{x}}=\dot{x}_{d}-\dot{x} \rightarrow 0$ is guaranteed.

\section{Appendix 2. Parameterized Linearization of the Dynamics/Kinematics}

To simplify the expressions of the dynamic parameters, the last link and the unknown tool can be treated as an equivalent link since they are fixedly connected throughout. According to equivalence principle, parameters of the new link are given as:

$m_{3 e}=m_{3}+m_{e} ; \quad l_{c 3 e}=\sqrt{x_{3 e}^{2}+y_{3 e}^{2}} ; \quad \delta_{3 e}=\arctan \left(y_{3 e} / x_{3 e}\right)$

$x_{3 e}=\left(m_{3} l_{c 3}+m_{e}\left(l_{c e} \cos \left(\delta_{e}\right)+l_{3}\right)\right) / m_{3 e} ; \quad y_{3 e}=m_{e} l_{c e} \sin \left(\delta_{e}\right) / m_{3 e}$.

$\delta_{3 e}$ is assumed to be known as a constant value to properly simplify the linearization. Then the robot dynamic parameters $\theta_{d}=\left[\theta_{d 1}, \theta_{d 2} \ldots \theta_{d 6}, \theta_{d 7}\right]^{T}$ are selected as follows:

$\theta_{d 1}=m_{1} l_{c 1}^{2}+I_{1}+m_{2} l_{1}^{2}+m_{2} l_{c 2}^{2}+I_{2}+m_{3 e}\left(l_{1}^{2}+l_{2}^{2}+l_{c 3 e}^{2}\right)+I_{3 e} ;$

$\theta_{d 2}=m_{2} l_{c 2}^{2}+I_{2}+m_{3 e}\left(l_{2}^{2}+l_{c 3 e}^{2}\right)+I_{3 e} ; \quad \theta_{d 3}=m_{3 e} l_{c 3 e}^{2}+I_{3 e} ;$

$\theta_{d 4}=m_{2} l_{1} l_{c 2} ; \theta_{d 5}=m_{3 e} l_{1} l_{c 3 e} ; \theta_{d 6}=m_{3 e} l_{1} l_{2} ; \theta_{d 7}=m_{3 e} l_{2} l_{c 3 e}$.

The matrices $M(q), C(q, \dot{q})$ and the expression of $\theta_{d k}$ with its estimation results are not listed here for simplicity. The matrix $W_{d}(q, \dot{q}, t)$ and the dynamic regression matrix $Y_{\mathrm{d} r}\left(q, \dot{q}, \dot{q}_{r}, \hat{\ddot{q}}_{r}\right)$ can be computed based on (12) and (28) respectively. $\widehat{Y}_{\mathrm{d} r}\left(q, \dot{q}, \dot{q}_{r}, \hat{\vec{q}}_{r}, \hat{\theta}_{k}\right)$ can be obtained from (22).

The robot kinematic parameters $\theta_{k}=\left[\theta_{k 1}, \theta_{k 2} \ldots \theta_{k 6}\right]^{T}$ are given as: $\theta_{k 1}=l_{1}, \theta_{k 2}=l_{2}, \theta_{k 3}=l_{3}+l_{e} \cos \left(\delta_{e}\right), \theta_{k 4}=l_{e} \sin \left(\delta_{e}\right)$, $\theta_{k 5}=l_{2}\left(l_{3}+l_{e} \cos \left(\delta_{e}\right)\right), \theta_{k 6}=l_{2} l_{e} \sin \left(\delta_{e}\right)$. The kinematic regression matrix $Y_{k}(q, \dot{q})$ and the matrix $W_{k}(t)$ are obtained from (5) and (14), respectively. 